\title{
Silent Microembolism on Diffusion-Weighted MRI after Coil Embolization of Cerebral Aneurysms
}

\author{
Sook Young Sim, MD, PhD'1, Yong Sam Shin, MD, PhD²
}

Purpose: The purpose of this study was to investigate the frequency and risk factors of procedure-related thromboembolism on diffusion-weighted imaging (DWI) associated with aneurysmal coil embolization.

Materials and Methods: We prospectively evaluated 39 consecutive patients with a cerebral aneurysm with DWI after coil embolization. All hyperintense lesions on DWI with a drop of apparent diffusion coefficient values were classified into acute thromboembolic infarction (larger than $5 \mathrm{~mm}$ in maximal diameters, and located in the vascular territory of the parent artery) and silent microembolism (single or multiple tiny dot-like lesion, less than $5 \mathrm{~mm}$, usually 1-2 $\mathrm{mm}$ in size). Possible risk factors for thromboembolic events included vascular risk factors, aneurysmal factors, and procedure-related factors.

Results: Hyperintense lesions on DWI were seen in 17 (43.6\%) patients and symptomatic DWI positive lesions were four (10.3\%). Acute thromboembolic infarction was observed in seven (17.9\%) patients and silent microembolism in $14(35.9 \%)$ patients. Numbers of silent microembolism ranged from 1 to 15 (mean: 2.86, standard deviation: 3.74). Silent microembolisms were located at ipsilateral $(n=3,21.4 \%)$, contralateral $(n=5,35.7 \%)$, bilateral $(n=4,28.6 \%)$, and not related $(n=2,14.3 \%)$ to the procedure site. There were no statistical significant risk factors in acute thromboembolic infarction. However, incidence of silent microembolisms was significantly correlated with left side approach (odds ratio, 4.44, 95\% confidence interval, 1.08-18.36; $P=0.03$ ).

Conclusion: Left side approach may have increased the likelihood of asymptomatic multiple scattered microemboli after aneurysmal coiling procedures. Particular care must be taken in the handling of guiding catheters, especially when proving left side great vessels.

Key Words : Diffusion Magnetic resonance imaging; Embolization; Therapeutic; Intracranial aneurysm; Thromboembolism

'Department of Neurosurgery, Seoul Paik Hospital, Inje University College of Medicine, Seoul, Korea

2Department of Neurosurgery, Seoul St. Mary's Hospital, The Catholic University of Korea, Seoul, Korea

Received March 19, 2012; accepted after revision June 25, 2012.

Correspondence to: Yong Sam Shin, MD, PhD, Department of Neurosurgery, Seoul St. Mary's Hospital, The Catholic University of Korea, 505 Banpo-dong, Seocho-gu, Seoul 137-701, Korea.

Tel. 82.2.2258.6125 Fax. 82.2.594.4248 E-mail: nsshin@catholic.ac.kr

This is an Open Access article distributed under the terms of the Creative Commons Attribution Non-Commercial License (http://creativecommons.org/licenses/by-nc/3.0) which permits unrestricted non-commercial use, distribution, and reproduction in any medium, provided the original work is properly cited. 
Since the novel endovascular approach for the intracranial aneurysms were introduced $[1,2]$, coil embolization plays an important role in treatment of cerebral aneurysms. The recent development of many devices and technique significantly improved the outcome of endovascular treatment of intracranial aneurysms, but the thromboembolic events still remain one of the most frequent and serious complications of coil embolization. The incidence of thromboembolic events resulting from conventional [3-8] and device assisted [8-10] coil embolization procedures is well established. However, those data were based on clinical and angiographic findings. Since the usefulness of diffusion-weighted imaging (DWI) immediate after the coil embolization of the aneurysms for detection of ischemic lesions was proposed [3], it has been well known that multiple small silent lesions can be observed on postoperative DWI after coil embolization $[6,7,9,10]$. While the frequency of the symptomatic territorial ischemic lesions and multiple scattered microembolism was evaluated in several small and a large series, the possible risk factors associated ischemic lesions after coil embolization of the intracranial aneurysms have not been fully analyzed. Some possible risk factors have been proposed. However, it was a retrospective study [10] or small prospective study limiting to unruptured aneurysms [6]. Therefore, we tried to evaluate whether any demographic, anatomic or technical factors contributed to an increased risk of thromboembolic events during these procedures in prospective series.

\section{MATERIALS AND METHODS}

\section{Patients Population}

We prospectively evaluated 39 consecutive patients with a cerebral aneurysm with DWI after coil embolization. Informed consent was obtained from all patients and legal representative. Eleven patients were male and 28 were female. The patients' ages ranged from 36 to 80 years (mean: 56.8 years, median: 56 years, standard deviation (SD): 11.7 years). Thirty-two aneurysms were located in the anterior circulation (posterior communicating artery-internal carotid artery junction, $n=14$; anterior communicating artery (AcomA), $n=9$; internal carotid artery-paraclinoid/ paraophthalmic segment, $\mathrm{n}=5$; middle cerebral artery (MCA), $n=3$; internal carotid artery bifurcation, $n=1$ ) and seven were in the posterior circulation (vertebral artery, $n=4$; top of the basilar artery, $n=3$ ). Thirty aneurysms were small $(<10 \mathrm{~mm})$ and nine were large
(10-25 mm). Except for the twelve aneurysms located on the midline, 15 lesions were on the right side and twelve were on the left. Twenty-six patients presented with acute subarachnoid hemorrhage (Hunt and Hess grade $\mathrm{I}, \mathrm{n}=1$; grade II, $\mathrm{n}=14$; grade III, $\mathrm{n}=9$; grade IV, $\mathrm{n}=1$; grade $\mathrm{V}, \mathrm{n}=1$ ). The Fisher grade [11] was distributed as follows: Fisher grades I, II, III, and $\mathbb{N}$ in 15 (38.5\%), 7 (17.9\%), $13(33.3 \%)$, and $4(10.3 \%)$ cases, respectively.

\section{Embolization Procedure}

Thirty-nine endovascular procedures were performed in 39 patients with one aneurysm each.

In all cases, cerebral angiography was performed by using a standard transfemoral approach to evaluate the morphologic features of the aneurysm, its anatomic relationship to parent artery and the presence of collateral circulation before the procedure.

Twenty patients had general anesthesia and 19 procedures were conducted with the patient under intravenous sedation. After gaining arterial access and placement of a $6 \mathrm{~F}$ guiding catheter, patients received an intravenous bolus of $100 \mathrm{IU} / \mathrm{kg}$ heparin followed by continuous infusion of $1,000 \mathrm{IU} / \mathrm{hr}$ to keep the activated clotting time at approximately at $2-2.5$ times the baseline. In selected cases, heparinization was continued for up to 24 hours with a target partial thromboplastin time of 60 to 90 seconds. In most patients with ruptured aneurysms, systemic heparinization was withheld or delayed until after successful placement of one or more coils. In all patients, the pressurized flush with 3,000 IU heparin/L saline for the guiding catheter and microcatheter was maintained and closely monitored for its fast drip. After control angiography for a working projection, the microcatheter was introduced through the guiding catheter and navigated into the aneurysm by using a fluoroscopic road map. Then, coil embolization was performed under fluoroscopic guidance using standard techniques. In the cases of wide-neck aneurysms, various techniques were required as a balloon remodeling in two patients, double catheter technique in two patients, and stent assisted embolization in one patient.

Serial angiographic runs were performed after placement of each coil or two, and at the final stage of the procedure to assess the degree of aneurysm obliteration, patency of parent artery, and thromboembolism. Thrombus or emboli, if present, were treated with fibrinolytic agents at the discretion of the surgeon. Antiplatelet agents were prescribed to selected patients with unruptured aneurysm as $100 \mathrm{mg}$ aspirin plus 75 
mg clopidogrel for three days prior to the procedure and for one month thereafter.

\section{Post Procedural Clinical and Radiological Assessment}

The degree of aneurysmal occlusion was classified as complete occlusion (no filling of aneurysm), nearly complete occlusion (minor contrast filling of aneurysm or small neck remnant), or partial occlusion. Each patient underwent a neurologic examination before, immediately after treatment, and during hospitalization.

All patients underwent postprocedural MRI within 48 hours postoperatively, which includes axial T1weighted, T2-weighted, fluid-attenuated inversion recovery and DWI. The parameters of the DWI were as follows: $b$ values, 0 and $1,000 \mathrm{~s} / \mathrm{mm}^{2}$; TR/TE, 10,000/
92.50; matrix, $128 \times 128$; field of view, $19 \times 19 \mathrm{~cm}$; section thickness, $7 \mathrm{~mm}$; and intersection gap, $0 \mathrm{~mm}$. If hyperacute ischemic lesions on DWI were noticed, the apparent diffusion coefficient (ADC) values in the corresponding region were correlated with the findings. Then, we identified the size, the numbers, and location of the lesions including vascular territories. All hyperintense lesions on DWI with a drop of ADC values were classified into acute thromboembolic infarction (larger than $5 \mathrm{~mm}$ in maximal diameters, and located in the vascular territory of the parent artery) and silent microembolism (single or multiple tiny dotlike lesion, less than $5 \mathrm{~mm}$, usually $1-2 \mathrm{~mm}$ in size, Fig. 1).

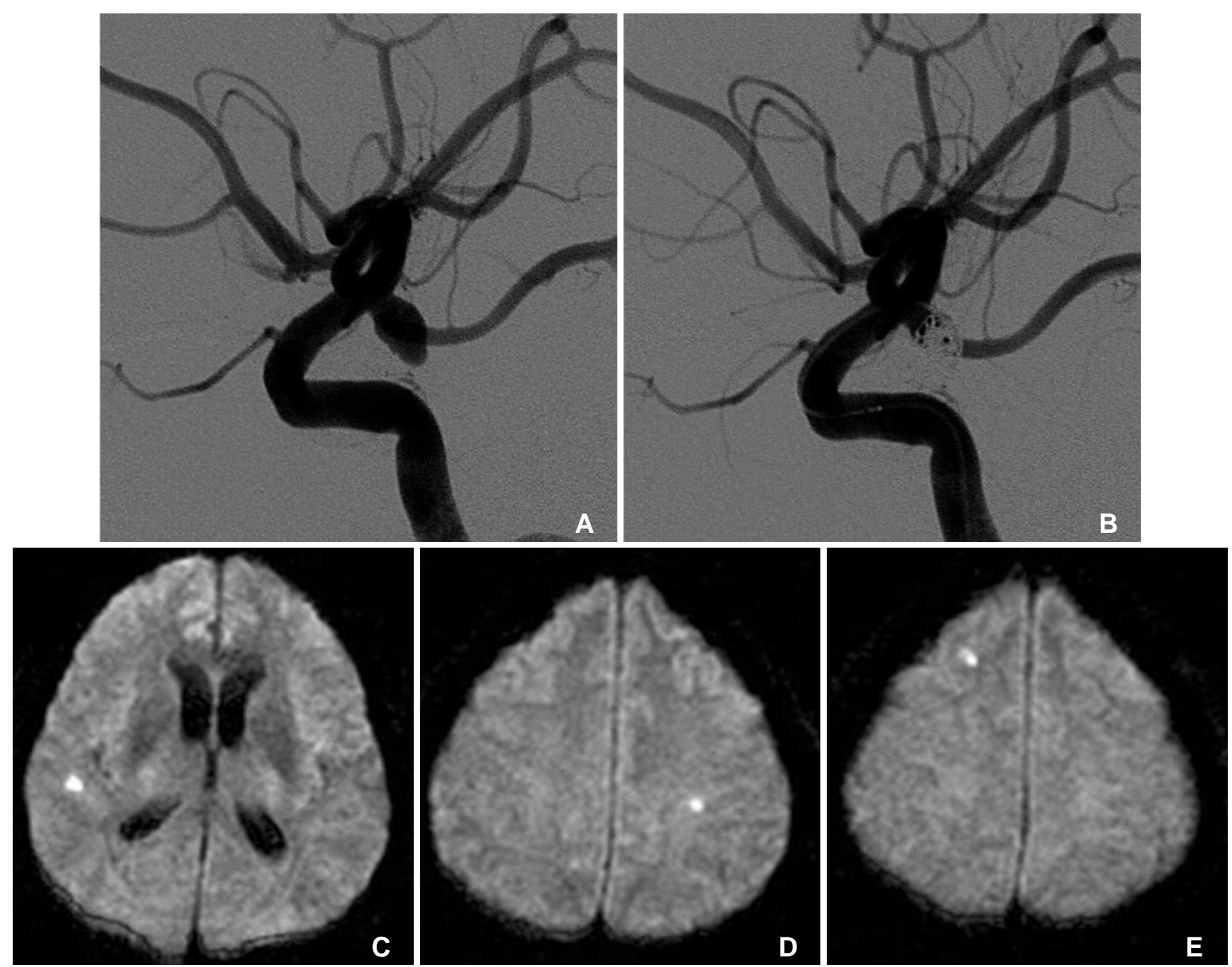

Fig. 1. A 55-year-old male with a ruptured posterior communicating artery (PComA) aneurysm.

A. Preoperative left internal carotid artery lateral angiograms.

B. Immediate postembolization image shows partial aneurysmal occlusion with a neck remnant around the right PComA orifices.

C-E. Diffusion-weighted images obtained 24 hours after the procedure shows multiple small hyperintense signals on ipsilateral and contralateral side of the procedures without any symptom. 


\section{Risk Factor analysis}

Possible risk factors for thromboembolic events included as below:

1) Vascular risk factors such as history of hypertension, smoking, diabetes mellitus, past ischemic stroke, total cholesterol level, as well as demographic factors including age and sex

2) Aneurysmal factors such as Hunt and Hess grade, Fisher grade, aneurysm location, size (maximal diameter, dome-to-neck ratio) and morphology (saccular, multi-lobulated, fusiform), atherosclerosis and vascular tortuosity of proximal vessels, flow type of aneurysm (bifurcation aneurysm, side wall aneurysm)

3) Procedure related factors such as method of anesthesia, perioperative antiplatelet or anticoagulant medication, total number of used coil, use of special embolization technique (balloon- or stentassisted technique, multiple microcatheter technique)

\section{Statistical analysis}

Univariate associations were assessed with frequency tables, Pearson's $\boldsymbol{x}^{2}$ tests for two independent proportions and linear by linear association for three or more independent proportions. Means were compared through the use of $t$-tests. Univariate odds ratio and $95 \%$ confidence interval was calculated to assess predictors of thromboembolic event in aneurysmal coiling procedures. Logistic regression was not performed because of the small sample size and the lack of multiple significant variables. SPSS (SPSS Version 17.0 for Windows, SPSS Inc., Chicago, IL, USA) was used for all analyses.

\section{RESULTS}

Complete obliteration, near-complete occlusion, and partial occlusion of the aneurysms were achieved in 24 (61.5\%), $11(28.2 \%)$, and $4(10.3 \%)$ patients, respectively. Two aneurysms ruptured during procedures due to microcatheter or coil penetration outside the aneurysms, all of which resulted in a minimal leak without clinical worsening. Hyperintense lesions on DWI were seen in 17 (43.6\%) patients and symptomatic DWI positive lesions were four (10.3\%). Acute thromboembolic infarction was observed in seven (17.9\%) patients and silent microembolism in 14 (35.9\%) patients. Among them, four patients had acute thromboembolic infarction and silent microembolism simultaneously. All microembolism were asympto- matic whereas four of seven acute thromboembolic infarction had clinical symptoms corresponding the ischemic lesions. In one patient with a ruptured posterior communicating artery aneurysm, intraprocedural thrombus developed in M3 branch and immediately resolved with 100,000 IU of intra-arterial urokinase. However, the patient had $47 \mathrm{~mm}$ sized hyperintense lesion in ipsilateral MCA territory on postoperative DWI with mild deficit at discharge. In two patients with ruptured vertebral dissection, sacrifice of the parent artery was inevitable resulting in lateral medullary infarction in both cases. Postprocedural DWI revealed a hyperintense lesion on lateral medulla in each patient and they suffered from transient lateral medullary syndrome. The other patient with a ruptured AcomA aneurysm had bilateral anterior cerebral artery (ACA) territory infarction on postoperative DWI, however, it appeared to be strongly related to vasospasm.

Numbers of silent microembolism ranged from 1 to 15 (mean: 2.86, median: 1, SD: 3.74). Silent microembolisms were located at ipsilateral $(n=3,21.4 \%)$, contralateral $(n=5,35.7 \%)$, bilateral $(n=4,28.6 \%)$, and not related $(n=2,14.3 \%)$ to the procedure site (Table 1$)$. Vascular territories of the silent microembolism included ACA ( $n=3,21.4 \%)$, MCA $(n=4,28.6 \%)$, posterior cerebral artery $(\mathrm{n}=1,7.1 \%)$, and borderzone $(n=3,21.4 \%)$. There was no statistically significant difference in each location related to the procedure and in each vascular territories $(P=0.29$ and $P=0.56$, respectively). Univariate analyses with the potential thromboembolic risk factors associated with endovascular procedures were separately conducted for acute

Table 1. Anatomical Distribution of the Silent Microembolism

\begin{tabular}{lcr}
\hline & Frequency & $\%$ \\
\hline Location related to the procedure & & \\
Ipsilateral & 3 & 21.4 \\
Contralateral & 5 & 35.7 \\
Bilateral & 4 & 28.6 \\
Not related & 2 & 14.3 \\
\hline Total & 14 & 100.0 \\
\hline Vascular territories & & \\
ACA & 6 & 42.9 \\
MCA & 4 & 28.6 \\
PCA & 1 & 7.1 \\
Borderzone & 3 & 21.4 \\
\hline Total & 14 & 100.0 \\
\hline
\end{tabular}

Abbreviations: ACA, anterior cerebral artery; MCA, middle cerebral artery; PCA, posterior cerebral artery 


\section{Silent Microembolism after Aneurysmal Coil Embolization}

Table 2. Univariate Analysis of Demographic and Clinical Characteristics of the Patients With and Without Multiple Scattered Microembolisms on DWI after Aneurysmal Coiling

\begin{tabular}{|c|c|c|c|c|c|c|}
\hline Variables & Total & $\begin{array}{c}\text { Microembolism (+) } \\
\text { on DWI }\end{array}$ & $\begin{array}{c}\text { Microembolism (-) } \\
\text { on DWI }\end{array}$ & Odds ratio & $95 \% \mathrm{Cl}$ & $P$-value \\
\hline Female, $\mathrm{n}(\%)$ & $28(72.8)$ & $9(64.3)$ & $19(76.0)$ & 0.57 & $0.14-2.37$ & 0.44 \\
\hline Age, mean, y & $56.79 \pm 11.67$ & $57.14 \pm 11.39$ & $56.60 \pm 12.05$ & & $-8.54-7.46$ & 0.89 \\
\hline \multicolumn{7}{|l|}{ Vascular risk factors } \\
\hline Hypertension, n (\%) & 19 (48.7) & $6(42.9)$ & $13(52.0)$ & 0.69 & $0.19-2.59$ & 0.58 \\
\hline DM, n (\%) & $2(5.1)$ & $1(7.1)$ & $1(4.0)$ & 1.85 & $0.11-32.01$ & 0.67 \\
\hline Smoking, n (\%) & $8(20.5)$ & $4(28.6)$ & $4(16.0)$ & 2.1 & $0.43-10.17$ & 0.35 \\
\hline Cholesterol, mean, mg/dl & $175.41 \pm 41.55$ & $177.07 \pm 48.26$ & $174.48 \pm 38.33$ & & $-31.06-25.87$ & 0.86 \\
\hline Previous stroke, $\mathrm{n}(\%)$ & $8(20.5)$ & $2(14.3)$ & $6(24.0)$ & 0.53 & $0.09-3.06$ & 0.47 \\
\hline \multicolumn{7}{|l|}{ Aneurysmal factors } \\
\hline Rupture, n (\%) & $26(66.7)$ & $10(71.4)$ & $16(64.0)$ & 1.41 & $0.34-5.81$ & 0.64 \\
\hline Hunt-Hess grade, $n(\%)$ & & & & & & 0.64 \\
\hline I & $1(3.8)$ & $0(0)$ & $1(100)$ & & & \\
\hline$\|$ & $14(53.8)$ & $6(42.9)$ & $8(57.1)$ & & & \\
\hline III & $9(34.6)$ & $3(33.3)$ & $6(66.7)$ & & & \\
\hline IV & $1(3.8)$ & $1(100)$ & $0(0)$ & & & \\
\hline V & $1(3.8)$ & $0(0)$ & $1(100)$ & & & \\
\hline Fisher grade, $\mathrm{n}(\%)$ & & & & & & 0.78 \\
\hline 1 & $15(38.5)$ & $4(26.7)$ & $11(73.3)$ & & & \\
\hline$\|$ & $7(17.9)$ & $3(42.9)$ & $4(57.1)$ & & & \\
\hline III & $13(33.3)$ & $5(38.5)$ & $8(61.5)$ & & & \\
\hline IV & $4(10.3)$ & $2(50.0)$ & $2(50.0)$ & & & \\
\hline Location, n (\%) & & & & & & 0.30 \\
\hline ICA & $20(51.3$ & $6(30.0)$ & $14(70.0)$ & & & \\
\hline ACA & $9(23.1)$ & $5(55.6)$ & $4(44.4)$ & & & \\
\hline MCA & $3(7.7)$ & $0(0)$ & $3(100)$ & & & \\
\hline Posterior circulation & $7(17.9)$ & $3(42.9)$ & $4(57.1)$ & & & \\
\hline Side, $\mathrm{n}(\%)$ & & & & & & 0.11 \\
\hline Right & $15(38.5)$ & $2(13.3)$ & $13(86.7)$ & & & \\
\hline Left & $12(30.8)$ & $5(41.7)$ & $7(58.3)$ & & & \\
\hline Midline & $12(30.8)$ & $7(58.3)$ & $5(41.7)$ & & & \\
\hline Configuration, n (\%) & & & & & & 0.82 \\
\hline Saccular & $29(74.4)$ & $10(34.5)$ & $19(65.5)$ & & & \\
\hline Multilobulated & $6(15.4)$ & $2(33.3)$ & $4(66.7)$ & & & \\
\hline Fusiform & $4(10.3)$ & $2(50.0)$ & $(50.0)$ & & & \\
\hline Diameter, mean, mm & $7.87 \pm 4.45$ & $8.29 \pm 4.81$ & $7.63 \pm 4.32$ & & $-3.73-2.41$ & 0.66 \\
\hline Dome/neck ratio, mean & $2.18 \pm 0.86$ & $2.49 \pm 1.10$ & $2.02 \pm 0.69$ & & $-1.30-0.35$ & 0.24 \\
\hline Side wall aneurysm, $\mathrm{n}(\%)$ & $22(56.4)$ & $8(57.1)$ & $14(56.0)$ & 1.05 & $0.28-3.92$ & 0.95 \\
\hline \multicolumn{7}{|l|}{ Procedural factors } \\
\hline Left side approach, $\mathbf{n}(\%)^{*}$ & $19(48.7)$ & $10(71.4)$ & $9(36.0)$ & 4.44 & $1.08-18.36$ & 0.03 \\
\hline Atherosclerosis, n (\%) & $8(20.5)$ & $3(21.4)$ & $5(20.0)$ & 1.09 & $0.22-5.45$ & 1.00 \\
\hline Tortuous curvature, $\mathrm{n}(\%)$ & $4(10.3)$ & $1(7.1)$ & $3(12.0)$ & 0.56 & $0.05-6.00$ & 0.63 \\
\hline IV anesthesia, n (\%) & $19(48.7)$ & $9(64.3)$ & $10(40.0)$ & 2.70 & $0.70-10.47$ & 0.15 \\
\hline Premedication, n (\%) & $9(23.1)$ & $2(14.3)$ & $7(28.0)$ & 0.43 & $0.08-2.43$ & 0.33 \\
\hline Heparinization, n (\%) & $17(43.6)$ & $5(35.7)$ & $12(48.0)$ & 0.60 & $0.16-2.31$ & 0.46 \\
\hline Postmedication, n (\%) & $15(38.5)$ & $5(35.7)$ & $10(40.0)$ & 0.83 & $0.22-3.23$ & 0.79 \\
\hline Used coil numbers, mean & $4.35 \pm 2.34$ & $4.08 \pm 2.66$ & $4.50 \pm 2.19$ & & $-1.23-2.07$ & 0.61 \\
\hline Special technique, $\mathrm{n}(\%)$ & $5(12.8)$ & $1(7.1)$ & $4(16.0)$ & 0.40 & $0.04-4.02$ & 0.64 \\
\hline
\end{tabular}

Abbreviations: DWI, diffusion-weighted image; $\mathrm{Cl}$, confidence interval; DM, diabetes mellitus; IV, intravenous

* : is statistically significant in the result of $x^{2}$ test 
thromboembolic infarction, for silent microembolism, and for all DWI positive lesions including both lesions. There were no statistical significant risk factors in acute thromboembolic infarction and all DWI positive lesions (Data not shown). However, the incidence of silent microembolisms was significantly correlated with left side approach (odds ratio, 4.44, 95\% confidence interval, $1.08-18.36 ; P=0.03$ ). Among total 19 cases of left side approach (left common carotid artery, $n=15$ and left vertebral artery, $n=4$ ), ten cases (52.6\%; left common carotid artery: $n=8$, left vertebral artery: $n=2$ ) of silent microembolism were detected on DWI, whereas only four events out of 20 cases $(20 \%)$ occurred in right side approach (right common carotid artery: $n=3$, right vertebral artery: $n=1$ ). Table 2 lists the demographic and clinical characteristics of the patients with silent microembolisms on DWI and the control group. Both groups were comparable in terms of demographic data, and the aneurysmal anatomic factors, and procedural factors. The other possible risk factors including vascular risk factors, aneurysmal factors and procedural factors were not associated with the occurrence of silent microembolisms (Table 2).

\section{DISCUSSION}

Although the ischemic events related to aneurysm coiling have been markedly decreased during last two decades, endovascular surgeons cannot exclude the possibility of encountering thromboembolic events either during or after an uneventful embolization procedure. The prevalence of thromboembolic complications, including transient ischemic attack (TIA) and stroke in early period of endovascular therapy, had been varied to be between $2.5 \%$ [12] to $21 \%$ [13]. As experience accumulated and the heparinization during and after the procedures became routine, these reported rates of TIA and stroke have decreased. However, the reported data only included thromboembolic ischemia with symptomatic and angiographically proven one.

Since the feasibility and the usefulness of DWI to detect ischemic brain changes in patients who had undergone endovascular treatment for an aneurysm have been proposed [3], frequency of thromboembolic events after aneurysmal coiling, even subclinical ones, can be estimated. Pelz et al. [5] have suggested that thromboembolic events related to aneurysmal embolization treatment may be more common than what has been reported in the literature. Several studies have been conducted to show the occurrence of postprocedural thromboembolic events after coil embolization using DWI for detecting early and small ischemic lesions [8]. These studies revealed that the incidence of procedure-related ischemic lesions on DWI were more frequent than expected, occurring in $10 \sim 69 \%$ [3, 6-10], and resulting in $5 \sim 26.9 \%$ of ischemic stroke with neurologic deficits $[4,5,7]$. Surprisingly, most of the DWI positive lesions were asymptomatic multiple microemboli. These results represent that absence of newly onset symptom after coil embolization does not mean perfect procedures without thromboembolic complications, thereby reawakening interest in the risk factors associated with these microemboli during endovascular procedures dealing with aneurysms for safer neurointervention [8].

There are many possible sources for embolic events during embolization treatment of cerebral aneurysms, including friable plaques and iatrogenic dissection in the parent vessels, air bubbles, and thrombus or fresh clots within aneurysms and catheters [5-7, 12, 14]. Mechanisms and risk factors of thrombus formation in a proximal arterial branch in which usually resulted in territorial infarction and need intraarterial thrombolysis have been reported as the partially thrombosed aneurysm in wide-necked aneurysm $[3,12]$, complications related to technical difficulties [12], thrombus formation from the catheter or guidewire, or herniation of the coils into the parent vessel [8]. There is a debate on whether procedures requiring adjunctive devices lead to an increased frequency of thromboembolic complications [7, 9, 15-17].

Review of literatures proposed the risk factors for symptomatic and asymptomatic abnormal signal on DWI as low levels of anticoagulation [5], distal emboli from initial catheterization or from clot at the aneurysmal neck $[3,5,7]$, larger aneurysm diameter $[4,8]$, and protruding loops of coils [4]. Use of adjunctive device such as balloon or complex procedure such as a double catheter technique had been reported to be associated with an increased risk of thromboembolic complication in several reports $[5,7,8]$, whereas in other reports showed the results that use of the balloon or other adjunctive device did not increase the risk of thromboembolism $[6,9,10]$.

In our prospective study, the risk of thromboembolic events during the treatment of intracranial aneurysms in patients with vascular risk factors was not more significant than those without risk factors. Aneurysmal anatomic and other procedural factors were not associated with an increased incidence of silent or symptomatic ischemia. The only variables found to influence this risk of silent multiple scattered microembolism 


\section{Silent Microembolism after Aneurysmal Coil Embolization}

during coil embolization was left side of approach via left common carotid artery and left vertebral artery. However, the observation of high incidence of microembolism after left side procedures does not mean that silent multiple microembolism is more frequent in left side hemisphere. It is very interesting that $79.6 \%$ of asymptomatic emboli were distributed contralateral, bilateral or not related to the procedural side given that all cases of symptomatic territorial infarction $(n=4)$ were distributed ipsilateral to the side of procedure corresponding the vascular territories of parent artery. Multiple microembolisms directly related to the procedure itself distal to the aneurysm were only found in $21.4 \%$. It is well known that multiple hyperintense lesions on DWI after coil embolization of the aneurysms were not always corresponding the vascular territory of the aneurysm's parent artery [3, 8]. Even though the possibility existed that microembolism might be originated from collateral flow from nontargeted vessels [8], the authors speculated that asymptomatic multiple microembolism might appear to be originated from the atherosclerotic plaques scraped off the aortic arch or great vessel wall by catheter manipulation [7], and left common carotid artery and left vertebral artery seem to be prone to risk of these complication due to the fact that left side great vessels, especially in left common carotid artery, are usually more difficult to probe because of its acute angle to the aortic arch. Although diagnostic angiography carries a very low risk $(0.07 \%)$ for developing permanent cerebrovascular complication [18], 17 in 66 (25.8\%) patients underwent diagnostic angiography showed DWI positive lesions in a pattern consistent with embolic events [14]. It is quiet difficult to conclude which step of the procedure-diagnostic angiography, initial guiding catheter manipulation, and coiling procedure- may play a role for most for thromboembolic events. Radiologic findings from this study suggest that most microembolism seems to be originated from proximal level of vasculatures at least below the superselection of the microcatheters. On the contrary, source of acute territorial thromboembolic infarction caused by fresh thrombus formation might be closely related to the coiling procedure itself.

This study has a limitation of small series compared to many variables and lack of preoperative control MRI. Nevertheless, it is a prospective study with consecutive patients dealing with new variables which have not been studied well in previous literatures. This is the first report to indicate that left side approach might increase the incidence of asymptomatic microembolism on DWI during aneurysmal occlusion by endovascular means.

DWI appears to be the most sensitive technique for acute thromboembolic events after coil embolization and seems to offer the ability to identify early progression of ischemic injury even if the patient has no symptom. Left side approach may have increased the likelihood of asymptomatic multiple scattered microemboli after aneurysmal coiling procedures. Particular care must be taken in the handling of guiding catheters, especially when proving left side great vessels from aortic arch. Further study is needed to evaluate the risk factors in a larger series.

\section{Acknowledgement}

The authors have no financial interest in any materials or device described in this article.

\section{References}

1. Guglielmi G, Vinuela F, Sepetka I, Macellari V. Electrothrombosis of saccular aneurysms via endovascular approach. Part 1: Electrochemical basis, technique, and experimental results. J Neurosurg 1991;75:1-7

2. Guglielmi G, Vinuela F, Dion J, Duckwiler G. Electrothrombosis of saccular aneurysms via endovascular approach. Part 2: Preliminary clinical experience. J Neurosurg 1991;75:8-14

3. Biondi A, Oppenheim C, Vivas E, Casasco A, Lalam T, Sourour $\mathrm{N}$, et al. Cerebral aneurysms treated by Guglielmi detachable coils: evaluation with diffusion-weighted MR imaging. AJNR Am J Neuroradiol 2000;21:957-963

4. Derdeyn CP, Cross DT, 3rd, Moran CJ, Brown GW, Pilgram TK, Diringer MN, et al. Postprocedure ischemic events after treatment of intracranial aneurysms with Guglielmi detachable coils. $J$ Neurosurg 2002;96:837-843

5. Pelz DM, Lownie SP, Fox AJ. Thromboembolic events associated with the treatment of cerebral aneurysms with Guglielmi detachable coils. AJNR Am J Neuroradiol 1998;19:1541-1547

6. Rordorf G, Bellon RJ, Budzik RE, Jr., Farkas J, Reinking GF, Pergolizzi RS, et al. Silent thromboembolic events associated with the treatment of unruptured cerebral aneurysms by use of Guglielmi detachable coils: prospective study applying diffusionweighted imaging. AJNR Am J Neuroradiol 2001;22:5-10

7. Soeda A, Sakai N, Murao K, Sakai H, Ihara K, Yamada N, et al. Thromboembolic events associated with Guglielmi detachable coil embolization with use of diffusion-weighted MR imaging. Part II. Detection of the microemboli proximal to cerebral aneurysm. AJNR Am J Neuroradiol 2003;24:2035-2038

8. Soeda A, Sakai N, Sakai H, Iihara K, Yamada N, Imakita S, et al. Thromboembolic events associated with Guglielmi detachable coil embolization of asymptomatic cerebral aneurysms. Evaluation of 66 consecutive cases with use of diffusion-weighted MR imaging. AJNR Am J Neuroradiol 2003;24:127-132

9. Albayram S, Selcuk H, Kara B, Bozdag E, Uzma O, Kocer N, et al. Thromboembolic events associated with balloon-assisted coil embolization: evaluation with diffusion-weighted MR imaging. AJNR Am J Neuroradiol 2004;25:1768-1777 


\section{Sook Young Sim, et al.}

10. Brooks NP, Turk AS, Niemann DB, Aagaard-Kienitz B, Pulfer K, Cook T. Frequency of thromboembolic events associated with endovascular aneurysm treatment: retrospective case series. $J$ Neurosurg 2008;108:1095-1100

11. Fisher CM, Kistler JP, Davis JM. Relation of cerebral vasospasm to subarachnoid hemorrhage visualized by computerized tomographic scanning. Neurosurgery 1980;6:1-9

12. Vinuela F, Duckwiler G, Mawad M. Guglielmi detachable coil embolization of acute intracranial aneurysm: perioperative anatomical and clinical outcome in 403 patients. J Neurosurg 1997;86:475-482

13.Martin D, Rodesch G, Alvarez H, Lasjaunias P. Preliminary results of embolisation of nonsurgical intracranial aneurysms with GD coils: the 1st year of their use. Neuroradiology 1996;38 (Suppl 1): S142-150

14. Bendszus M, Koltzenburg M, Burger R, Warmuth-Metz M, Hofmann E, Solymosi L. Silent embolism in diagnostic cerebral angiography and neurointerventional procedures: a prospective study. Lancet 1999;354:1594-1597

15. Moret J, Cognard C, Weill A, Castaings L, Rey A. The "Remodeling technique" in the treatment of wide neck intracranial aneurysms. Angiographic results and clinical follow-up in 56 cases. Interv Neuroradiol 1997;3:21-35

16. Malek AM, Halbach VV, Phatouros CC, Lempert TE, Meyers PM, Dowd CF, et al. Balloon-assist technique for endovascular coil embolization of geometrically difficult intracranial aneurysms. Neurosurgery 2000;46:1397-1406

17. Nelson PK, Levy DI. Balloon-assisted coil embolization of widenecked aneurysms of the internal carotid artery: medium-term angiographic and clinical follow-up in 22 patients. AJNR Am J Neuroradiol 2001;22:19-26

18. Cloft HJ, Joseph GJ, Dion JE. Risk of cerebral angiography in patients with subarachnoid hemorrhage, cerebral aneurysm, and arteriovenous malformation: a meta-analysis. Stroke 1999;30: $317-320$ 